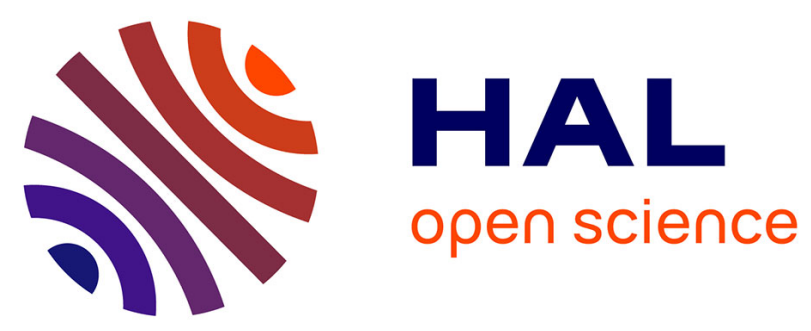

\title{
Epidemics in Venice: On the Small or Large Nature of the Pre-modern World
}

\author{
Giovanni Colavizza
}

\section{To cite this version:}

Giovanni Colavizza. Epidemics in Venice: On the Small or Large Nature of the Pre-modern World. 2nd International Workshop on Computational History and Data-Driven Humanities (CHDDH), May 2016, Dublin, Ireland. pp.33-40, 10.1007/978-3-319-46224-0_4 . hal-01616300

\author{
HAL Id: hal-01616300 \\ https://hal.inria.fr/hal-01616300
}

Submitted on 13 Oct 2017

HAL is a multi-disciplinary open access archive for the deposit and dissemination of scientific research documents, whether they are published or not. The documents may come from teaching and research institutions in France or abroad, or from public or private research centers.
L'archive ouverte pluridisciplinaire HAL, est destinée au dépôt et à la diffusion de documents scientifiques de niveau recherche, publiés ou non, émanant des établissements d'enseignement et de recherche français ou étrangers, des laboratoires publics ou privés. 


\title{
Epidemics in Venice: on the Small or Large Nature of the pre-Modern World
}

\author{
Giovanni Colavizza \\ École Polytechnique Fédérale de Lausanne (EPFL), Switzerland
}

\begin{abstract}
Marvel et al. [12] recently argued that the pre-modern contact world was physically and, by set inclusion, socially not small-world. Since the Black Death and similar plagues used to spread in well-defined waves, the argument goes, the underlying contact network could not have been small-world. I counter here that small-world contact networks were likely to exist in pre-modern times in a setting of the greatest importance for the outbreak of epidemics: urban environments. I show this by running epidemic diffusion simulations on the transportation network of Venice, verifying how such network becomes small-world when we account for naval transportation. Large epidemic outbreaks might not have been even possible without the catalyst of urban small-worlds.
\end{abstract}

Keywords: Epidemics, Venice, Small-world networks

\section{Introduction}

Pre-modern epidemics still foster interest, especially around their etiology, dynamics and impact [2]. A key topic in this respect is understanding the mechanics of different kinds of epidemics. For example, the Black Death might have been an exceptional, if not completely different phenomenon from the bubonic plague [7]. Pre-modern plague outbreaks are generally considered to have been more impactful in cities than in the countryside, where the population density was lower and the means of transportation not as effective [1]. Evidence suggesting a strong link between trade routes and sequences of plague outbreaks, as well as their seasonality, has been suggested [6]. As a consequence, the interaction of different kinds of epidemics and the spatio-temporal dynamics of an outbreak in a urban environment might have been more complex than expected [8]. In view of the lack of extensive data sources on pre-modern epidemics, modeling efforts are of great importance in order to help bounding the limits of the possible.

A small-world effect is an observation of a low average shortest path length between any two nodes in a network, irrespective of the number of nodes in such a network and their average clustering coefficient [13]. In practice, usually a few edges connecting far-away nodes suffice to make the whole network small-world: an example are modern transportation networks, where flight routes among cities connect far-away urban ground networks. Despite small-world contact networks being intuitively linked with fast spreading epidemics, in practice spreading often slows down due to structural and temporal effects [9]. As an example, [10] found 
that small-world networks can present slow spreading due to topological effectsdense intra-cluster and sparse inter-cluster connectivity - and bursty activity patterns of individuals.

Marvel et al. [12] recently claimed that the contact network of the premodern world was not small-world - not physically nor socially. Their evidence is the wave-like representation of epidemic diffusion at a continental scale in premodern Europe, examples of which are to be found in most historical atlases. I contend here that it is likely that the pre-modern world was small-world, at least in urban centers. This is shown by running epidemic spread simulations on the transportation network of the city of Venice iterating over two steps: local SIR processes on fully mixed groups of persons interacting by random travelers. As soon as naval transportation is accounted for, the network becomes effectively small-world. This paper is organized as follows: methods are presented in Section 2 , namely the transportation network of Venice and the epidemic model; Section 3 describes the simulations and Section 4 concludes.

\section{Method}

A simulation is proposed in order to assess to what extent the small-world of physical contacts could be a pre-modern phenomenon in a urban setting. Taking the transportation network of the city of Venice as a case study, a realistic-size population is distributed in the city, according to the urban density of different areas. The population is split in small groups, corresponding to the nodes of the network, where full mixing is assumed and SIR processes are run independently. This small groups interact with each other via travelers, who can move by land or water.

\subsection{Venice Transportation Network}

The transportation network of Venice was manually constructed from the modern topography of Venice, from which land and sea routes have been exported as geographical referenced segments. Nodes are created at crossing vertices, or crossroads, mirroring the urban density of any given area of the city. Edges are classified as being on land ("calle" is Venetian, green in Fig. 1) or water ("canal", blue), while nodes are classified as connecting only water edges (cyan), only land edges (grey) or connecting at least one of each typology (red). The resulting network possess 7384 nodes, 8861 edges and a low average degree of 2.4 , typical of transportation networks. If we consider our proxy of urban density to be a good approximation for the density of population in a given area of the city, and if we reasonably assume that the topography of Venice did not substantially change from the XVI century to the present day, then our network can be used to simulate epidemic outbreaks in the city.

Evidently, Venice is circled by water and mixed nodes are just a lower-bound approximation of all possible landings in the city. Venice is also known for its exceptional condition of a "floating city", yet the availability of water routes 


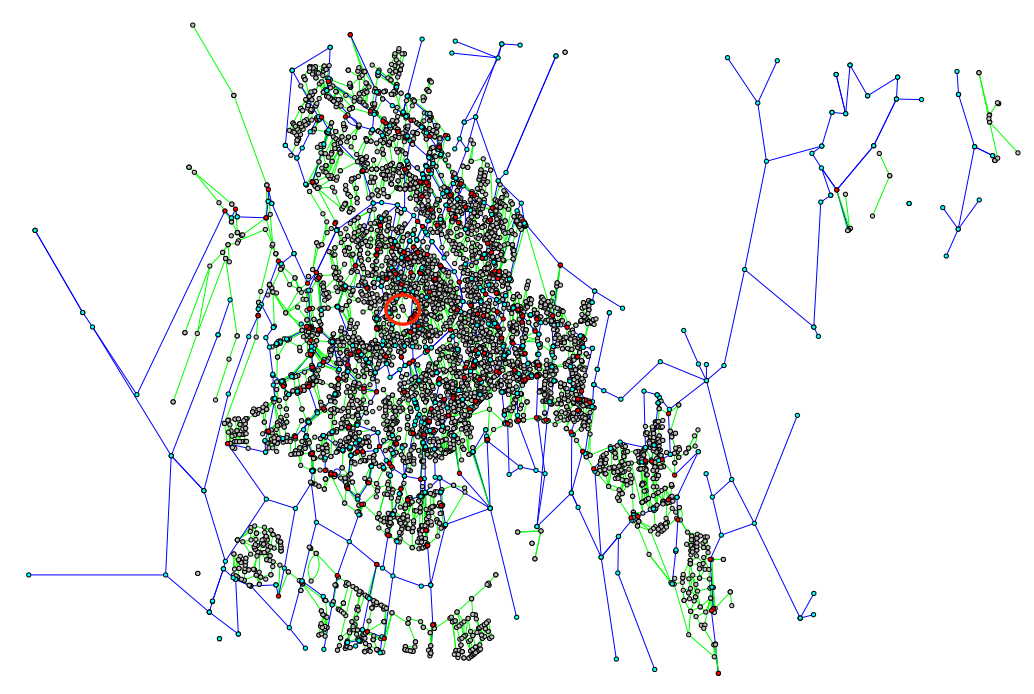

Fig. 1. The transportation network of Venice. Green edges are land routes and blue edges water routes; grey nodes connect only land edges, cyan nodes connect only water edges, and red nodes connect at least one edge per typology. The red circle indicates the starting point of epidemic simulations.

was a common feat of many pre-modern urban settlements. On a larger scale, even Europe is almost circled by water, and possesses several river basin areas. Nevertheless the extent to which Venice can be taken to be representative of premodern urban environments in this respect remains to be clarified. In order to account for Venice's alleged specificity, the simulations will be rather conservative with respect to the likelihood of naval travels.

If we reasonably assume Venice to have had a maximum of circa 150'000 inhabitants during the XV to XVIII centuries ${ }^{1}$, and assign it to all land nodes uniformly, we total 23 persons per node. The outbreak and spread of an epidemic can then be simulated by a discrete model which iterates between two selfconclusive phases (i.e. each phase is computed for all nodes independently before moving to the next phase):

- Step 1: the population of each node is considered to be fully-mixed and the time evolution of the infected fraction of the population within each node is modeled using standard approaches, here we adopt the SIR model.

- Step 2: a fraction of the population moves according to different functions (power law, exponential or geometrical progression).

\footnotetext{
${ }^{1}$ This is an approximation of Venetian population before the major plagues of the years 1576 and 1630-31. Estimates can be found e.g. in [5], p. 54. Historical analyses of the two plagues can be found in [15] and [17], respectively.
} 


\section{$2.2 \quad$ Epidemic Model}

The epidemic model is composed of three elements: a model for the development of the infection within each node, a traveling process and its associated travel probability function.

The susceptible $s$, infected $i$, recovered $r$ (or anyway removed) model (=SIR) prescribes to divide the population into three classes. Infected individuals have contact with susceptible individuals at an average rate of $\beta$ (contact rate), and infected individuals recover at an average rate of $\gamma$ (infection period). This model is thus governed by the following system of differential equations:

$$
\frac{d s}{d t}=-\beta \cdot s_{t} \cdot i_{t} ; \frac{d i}{d t}=\beta \cdot s_{t} \cdot i_{t}-\gamma \cdot i_{t} ; \frac{d r}{d t}=\gamma \cdot i_{t}
$$

with $s_{t}+i_{t}+r_{t}=1$ at all times. Default values of $\beta=0.05$ and $\gamma=0.1$ were used in all experiments. Note that in our setting these values need not be above the critical threshold for a transition to an epidemic regime, which is $\beta \geq \gamma$ with a fully mixed population [14].

After the population has been updated according to the SIR model, travel occurs. For each node independently:

1. An amount of traveling population is selected uniformly at random.

2. Each traveler randomly selects a neighboring land node with probability $p$ or, if it is in a red node, it randomly jumps to another red node with probability $1-p$. Naturally, $p=1$ if the traveler is in a grey node, and backwards movements are possible.

3. The number of movements done by each traveler is given by different travel probability functions. Given $p(d)$ the probability of traveling $d$ steps and $k$ a kernel factor, three functions have been tested: (i) the power law $p(k ; d)=$ $e^{-k \cdot d}$, (ii) the exponential $p(k ; d)=d^{-k}$ and (iii) a geometrical progression $p(d)=p(d-1) / 2$. A default kernel parameter of $k=2.0$ has been used.

The proposed model is an approximation and does not account for several aspects of the real-world phenomenon. One sensible limitation is the absence of real movement patterns which account for the relative importance of specific destinations, which could be integrated for example using a gravity model [16].

\section{Simulation}

The simplest way to simulate an epidemic outbreak is to infect a certain amount of persons in a random land node, where this amount needs to be sufficient for some traveler to survive and move. A starting condition of one node with three infected individuals was enough to generate an epidemic outbreak. For the purpose of this paper the origin of the epidemics does not matter, provided the randomly selected node is connected to the principal component of the transportation network. For what follows a node in the "San Polo" parish has been selected as the starting point (red circled in Figure 1). 
In order to follow the spread of the epidemic, we use two measures calculated at any time step $t$. First, the standard Infection Rate $I R(t)$, the proportion of infected persons at any give time $t$ over all nodes, defined as:

$$
I R(t)=\frac{\sum_{v=1}^{n} i\left(v_{t}\right)}{\sum_{v=1}^{n}\left[i\left(v_{t}\right)+s\left(v_{t}\right)\right]}
$$

Secondly, a customized version of the Basic Reproduction Number, called transmissibility, largely inspired by [18]. This transmissibility $T R(t)$ is an approximated lower-bound to the average probability of a previously uninfected node to receive an infected traveler at time $t$. Define $V$ to be the set of nodes in the transportation network, $N(v)$ to be the distance-1 neighborhood of node $v$ and $\operatorname{Ti}\left(v_{t}\right)$ the number of infected travelers of node $v$ at time $t$, then:

$$
T R(t) \approx \frac{1}{|V|} \sum_{v \in V: i(v)=0}\left\{\frac{\sum_{u \in N(v)} 1-\left[\frac{|N(u)-1|}{|N(u)|}\right]^{T i\left(u_{t}\right)}}{|N(v)|}\right\}
$$

Transmissibility is approximated because it only considers adjacent nodes i.e. travels of distance 1, and a lower-bound to the real measure given the division by the total number of nodes in the network. The resulting measure is a trend of the infection, strictly correlated with the Infection Rate and anticipating it.

The results of simulations are summarized in Figures 2(a) and 3(a) for the scenario where $p=1$ (thus naval travel probability is 0 ). Figures 2(b) and 3(b) show the scenario with $p=0.85$ and thus naval travel probability is 0.15 . All simulations were run multiple times (blue lines) and averaged (red lines), over 200 time ticks. Note that the two settings correspond to using two different transportation networks: one where red nodes are not connected to other red nodes, and only land routes are open; another where red nodes are connected with all other red nodes in the network. The former network has an average path length of 34 and a diameter of 91, the latter has an average path length of 8.6 and a diameter of 35. Simulations have been run with a power law travel function: both exponential and geometrical progression do not change the outcomes of the two simulations, only their timings (an exponential functions lags the spread, a geometrical progression accelerates it).

If we disallow for naval travels, we clearly see the wave-like epidemic spreading process taken by Marvel et al. [12] as evidence for the lack of a small-world contact network in the population. In particular, the outcome is a low-infection process, shown in Figure 2(a), which behaves according to the topology of the network as the front of the epidemics progresses, Figure 3(a). This model is clearly sensible to the topology of the network. For example the rise in transmissibility from ticks 40 to 50 is due to the spread reaching the dense area before the "Rialto" bridge, and the second major peak after tick 100 is mainly due to the spread moving after the bottleneck of the "Rialto" bridge. Running the same 


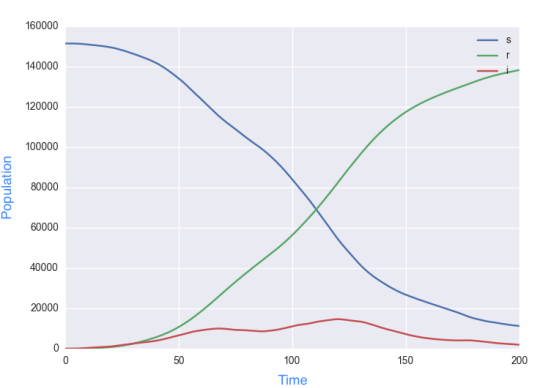

(a) No naval travel.

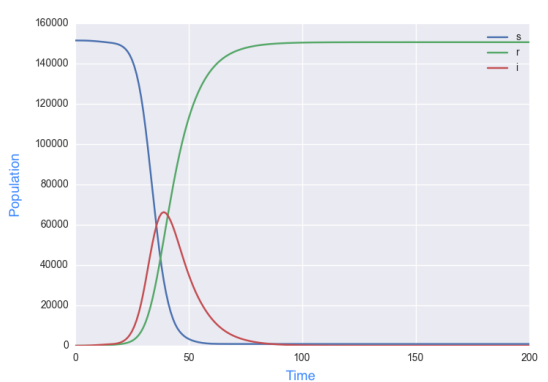

(b) Naval travel.

Fig. 2. SIR processes over the whole population, averaged over all simulation runs.

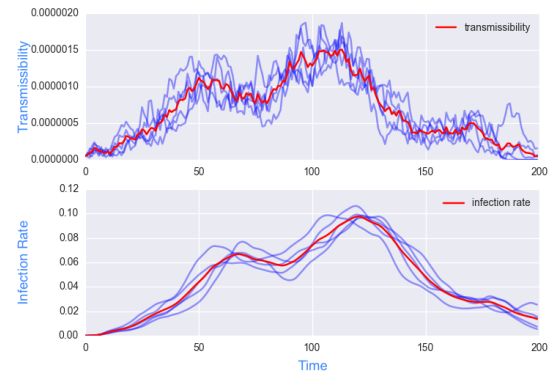

(a) No naval travel.

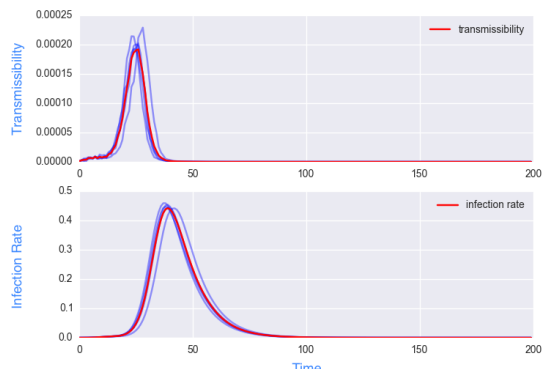

(b) Naval travel.

Fig. 3. Infection Rate and Transmissibility over time. The outcomes of several runs of the simulation (in blue) are averaged (in red) to show an estimation of empirical variance.

process on a regular square lattice of comparable size - details are omitted for brevity - produces a similarly steady process of wave-like diffusion with a monotonic increase of the Infection Rate until a global maximum is reached, followed by a steady decrease. The reason for this behavior is of course the regularity of the underlying topological network of the regular lattice, as results are consistent with previous literature on the topic [11].

Conversely, even a low probability of naval travel makes the process behave as in a small-world setting, with little to no effect of the underlying network topology, as shown in Figures 2(b) and 3(b). It is therefore likely that urban centers and water routes acted respectively as catalysts and fast carriers of epidemics in pre-modern times as they do now, in a sort of jump and burst pattern. The wave-like representation of pre-modern epidemic spreads across Europe might therefore be just an artifact of data aggregation. 
I thus suggest that an alternative model, with cities or urban conglomerates as supernodes in a network of land and water transportation, might better explain pre-modern epidemic outbreaks at the continental level. Each supernode, a city, should contain a fully-connected population of potential hosts (rats, humans, etc.). Eventually, according to seasonal patterns, cities communicate, mainly via water transport (either by sea or by rivers). The proposed approach bears strong resemblance to the super-networks of family households initially proposed in [3], and further expanded in [4], where every node in the supernetwork is a different network in itself-in our case small-world cities.

\section{Conclusions}

I investigated via epidemic spread simulation how the pre-modern world was likely to have been small-world at the urban scale, taking the transportation network of Venice as a case study. Without naval travel, the epidemic expands in waves, closely tied with the topology of the network. On the contrary, even with a low probability of naval travel accounted for, the process becomes qualitatively small-world. I thus argue that the interaction of small-world epidemics in cities, especially ports, and the jumps permitted by naval travels might account for the spread of large-scale epidemics in pre-modern times more than a generalized wave-like process induced by an exponentially decaying contact kernel. Several research questions remain open, specifically:

1. to what extend the case study of Venice can be taken to be representative of pre-modern urban environments in general?

2. Will the proposed model account for epidemic spread at a European scale?

3. Will evidence from historical records back the proposed model?

The purpose of this paper is two-fold. First, to caution in the use of aggregated evidence, as multiple interpretations can usually coexist with quite different mathematical models which account for the same measurable outcomes. Secondly, to suggest that the historical process of the reduction of distances across the globe, mainly thanks to transportation and communication technologies, has been gradual albeit increasingly accelerating. Small-world mechanics now proper to large-scale global networks might be found during pre-modern times in smaller-scale networks, such as urban environments. The quest then becomes to understand the pre-modern world as potentially being one where small and large-world mechanics coexisted at different times and scales.

\section{Acknowledgments}

I thank colleagues Isabella Di Lenardo and Olivier Delang for sharing the transportation network of Venice, and their students for making it. I also acknowledge EPFL students Joachim De Jonghe, Nicolas Duthilleul and Théo Lamaire for their preliminary efforts in the project. 


\section{References}

1. Alfani, G.: Il Grand Tour dei Cavalieri dell'Apocalisse. Marsilio (2010)

2. Alfani, G., Sansa, R.: Il ritorno della peste? Un'introduzione alla storiografia recente. Popolazione e Storia 2, 9-19 (2015)

3. Ball, F., Mollison, D., Scalia-Tomba, G.: Epidemics with two levels of mixing. Annals of Applied Probability 7, 46-89 (1997)

4. Ball, F., Neal, P.: Network epidemic models with two levels of mixing. Mathematical Biosciences 212(1), 69-87 (2008)

5. Beltrami, D.: Storia della popolazione di Venezia dalla fine del secolo XVI alla caduta della Repubblica. Cedam (1954)

6. Bossak, B.H., Welford, M.R.: Did medieval trade activity and a viral etiology control the spatial extent and seasonal distribution of Black Death mortality? Medical Hypothesis 72, 749752 (2009)

7. Christakos, G., Oleab, R., Yua, H.L.: Recent results on the spatiotemporal modelling and comparative analysis of Black Death and bubonic plague epidemics. Public Health 121, 700720 (2007)

8. Ell, S.: Three days in October of 1630: detailed examination of mortality during an early modern plague epidemic in Venice. Review of Infection Diseases 11(1), 128-141 (1989)

9. Holme, P.: Network reachability of real-world contact sequences. Physical Review E 71(4) (2005)

10. Karsai, M., Kivelä, M., Pan, R.K., Kaski, K., Kertész, J., Barabási, A.L., Saramäki, J.: Small but slow world: How network topology and burstiness slow down spreading. Physical Review E 83(2) (2011)

11. Keeling, M.J., Eames, K.T.: Networks and epidemic models. Journal of The Royal Society Interface 2(4), 295-307 (2005)

12. Marvel, S.A., Martin, T., Doering, C.R., Lusseau, D., Newman, M.E.J.: The smallworld effect is a modern phenomenon (2013)

13. Newman, M.E.J.: Networks: an introduction. Oxford University Press (2010)

14. Newman, M.E.J.: The spread of epidemic disease on networks. Physical Review E 66(016128) (2002)

15. Preto, P.: Peste e società a Venezia (1576). Neri Pozza (1984)

16. Simini, F., González, M.C., Maritan, A., Barabási, A.L.: A universal model for mobility and migration patterns. Nature 484, 96-100 (2012)

17. Ulvioni, P.: Il gran castigo di Dio: carestia ed epidemie a Venezia e nella Terraferma, 1628-1632. Franco Angeli (1989)

18. Witten, G., Poulter, G.: Simulations of infectious diseases on networks. Computers in Biology and Medicine 37, 195-205 (2007) 